\title{
Effects of mutual coupling on interference mitigation with a focal plane array
}

Michael A. Jensen

jensen@byu.edu

Karl F. Warnick

warnick@byu.edu

Follow this and additional works at: https://scholarsarchive.byu.edu/facpub

Part of the Electrical and Computer Engineering Commons

\section{Original Publication Citation}

Warnick, K. F., and M. A. Jensen. "Effects of Mutual Coupling on Interference Mitigation with a Focal Plane Array." Antennas and Propagation, IEEE Transactions on 53.8 (25): 249-8

\section{BYU ScholarsArchive Citation}

Jensen, Michael A. and Warnick, Karl F., "Effects of mutual coupling on interference mitigation with a focal plane array" (2005). Faculty Publications. 355.

https://scholarsarchive.byu.edu/facpub/355 


\title{
Effects of Mutual Coupling on Interference Mitigation With a Focal Plane Array
}

\author{
Karl F. Warnick, Senior Member, IEEE, and Michael A. Jensen, Senior Member, IEEE
}

\begin{abstract}
A focal plane array feed of electrically small elements has been proposed as a means for achieving high sensitivity for radio astronomy applications in the presence of radio frequency interference (RFI). For a broadband system, mutual coupling effects become significant as the array element spacing becomes small relative to the electromagnetic wavelength. We present a theoretical framework for modeling the effects of mutual coupling and for determining the optimal multiport matching network between array elements and front-end transistor low-noise amplifiers for maximum signal-to-noise ratio (SNR). Numerical results are given for a model scenario including spillover and amplifier thermal noise and point signal and interference sources. A suboptimal self-impedance matching network yields performance close to that of a full network match.
\end{abstract}

Index Terms-Antenna array mutual coupling, electromagnetic interference, radio astronomy.

\section{INTRODUCTION}

I NTERFERING signals radiated by ground-based, aircraft, and satellite-borne radio transmitters pose a threat to sensitive radio astronomical observations. Methods for mitigating radio frequency interference (RFI) include time and frequency blanking, adaptive cancellation and subtraction [1]-[3], and spatial filtering [4]-[8]. One approach to implementation of spatial filtering techniques is to replace large reflector antennas with arrays of many small elements [9]. Spatial filtering techniques have been explored in conjunction with arrays of medium size elements, including the Allen telescope array (ATA) [10] and the square kilometer array (SKA) [11].

Spatial filtering methods can also be implemented with a focal plane array feed in conjunction with a large reflector [12]. In communications and radar applications, array feeds have been used for many years to achieve shaped pattern footprints, electronically scanned beams, and compensation for reflector surface abberations [13]-[16]. Focal plane arrays for radio astronomy observations have advantages over aperture plane arrays in simpler signal processing and field of view [17]. Because of stringent sensitivity requirements, most practical array feeds for radio astronomy have employed electrically large, waveguide type feeds with each element individually matched to the reflector aperture and minimal or no signal

Manuscript received December 17, 2004; revised April 20, 2005. This work was supported in part by the National Science Foundation under an Advanced Technologies and Instrumentation Grant AST-0352705 and an Information Technology Research Grant CCR-0313056, and in part by the U.S. Army Research Office under the Multi-University Research Initiative (MURI) Grant W911NF-04-1-0224.

The authors are with the Department of Electrical and Computer Engineering, Brigham Young University, Provo, UT 84602 USA (e-mail: warnick@byu.edu).

Digital Object Identifier 10.1109/TAP.2005.852278 combining between elements [18], [19]. Arrays of more closely spaced, electrically small elements require complex signal processing but offer greater control over the far field beam pattern [20], [21]. Beams can either be formed electronically or synthesized from correlations [22]. Prototype arrays of this type for radio astronomy applications have been constructed and tested [23]-[26].

For a broadband array, mutual coupling becomes important at low frequencies due to reduced electrical element spacing. The impact of antenna coupling on focal plane array performance is well known in the radio astronomy community [18]. In a classic paper, Stein [27] analyzed mutual coupling in terms of element pattern overlap integrals and derived a bound on aperture efficiency. It is important to note that the Stein efficiency bound is based entirely on conservation of energy, so that if a mutually coupled array is matched for maximum power transfer to a load, the effective element patterns are orthogonal and the Stein efficiency becomes $100 \%$. Further progress in understanding mutual coupling has required consideration of the details of the elements and near field interactions. Coupling models are available for waveguide feed elements [28], Vivaldi antennas [29], and dielectric rod antennas [30]. For the case of an interferometric radiometer with small element spacings, a network theory analysis has been performed [31] to include the effects of coupling and amplifier noise. A similar framework has been developed for analyzing the impact of mutual coupling on the performance of multiantenna communications systems [32].

The aim of this paper is to develop a framework for treating mutual coupling effects for focal plane array feeds in the microwave range. This approach builds on the network analyzes of [31], [33] by including an impedance matching network between the coupled array and amplifiers. The framework is applied to a model problem consisting of a seven element dipole array and a $25-\mathrm{m}$ paraboloidal reflector. Particular attention is paid to the beamformer output signal-to-noise ratio (SNR), which is proportional to the system $G / T_{\text {sys }}$ (gain divided by system temperature). The simulations compare the performance for optimal and suboptimal matching networks over a broad operating bandwidth, with and without an interfering signal.

\section{ARray FeEd AND ReCEIVER Model}

The problem of interest in this analysis involves an array-fed reflector antenna operating as a receiver. A single plane wave representing the desired signal as well as plane waves created by interferers excite the system. Furthermore, spillover noise received by the antenna system (which is dependent on the feed spillover efficiency) as well as thermal noise generated in the front-end low-noise amplifiers (LNAs) corrupt the signal. For 
very low SNR applications such as radio astronomy, a signal is typically detected as a small perturbation relative to a temporally or spectrally averaged thermal noise power baseline. For this reason, the key system performance metric might be termed the signal to noise fluctuation ratio

$$
\mathrm{SNFR}=\frac{P_{\text {sig,out }}}{\Delta P_{\text {noise, out }}}
$$

where the powers are measured at the output port of a signalcombining network (beamformer). Assuming that the system gain is stable, the standard deviation of the thermal noise is

$$
\Delta P_{\text {noise }, \text { out }}=\frac{\alpha P_{\text {noise, out }}}{\sqrt{B t}}
$$

where $\alpha$ is on the order of unity and depends on the details of the detector, $B$ is the noise bandwidth, and $t$ is the integration time.

Interfering signals are likely to be temporally and spectrally nonstationary and therefore cannot be subtracted using a baseline approach. In the presence of RFI, the least optimistic performance metric is the signal to interference plus noise fluctuation ratio

$$
\mathrm{SINFR}=\frac{P_{\text {sig,out }}}{P_{\text {int,out }}+\Delta P_{\text {noise,out }}} .
$$

Because the noise standard deviation decreases with integration time, interference is less tolerable for long integration times. In this paper, for simplicity we choose a short integration window, such that $\alpha / \sqrt{B t}=1$, in which case SNFR and SINFR reduce to signal to noise ratio (SNR) and signal to interference plus noise ratio (SINR). The SNR is proportional to the system sensitivity $G / T_{\text {sys }}$, and the SINR is an effective system sensitivity if the interferer power is included in $T_{\text {sys }}$. The goal of this section is to characterize the signal, interferer, and noise contributions at the LNA outputs.

\section{A. Array Open-Circuit Response}

The first phase of the analysis is to determine the vectors of signal and interferer voltages and spillover noise covariance matrices at the antenna terminals. We will conduct our network analysis assuming that these quantities represent the antenna terminal voltages with all elements terminated in an open circuit. Because adjacent antennas that are open circuited have little impact on the radiation pattern of a driven antenna when the element spacing is reasonable, we will approximate the electric field radiation pattern for the $m$ th array element in the absence of the reflector, denoted here as $\bar{E}_{m}^{\text {inc }}$, by the isolated element pattern. The open circuit array element pattern overlap matrix is then given as

$$
\hat{A}_{m n}=\frac{1}{2 \eta P_{m}} \int \bar{E}_{m}^{\mathrm{inc}} \cdot \bar{E}_{n}^{\mathrm{inc}} r^{2} d \Omega
$$

where $\eta$ is the characteristic impedance of free space, $P_{m}$ is the total power radiated by the $m$ th element, and $r$ is the (arbitrary) distance at which the radiated fields $\bar{E}_{n}^{\text {inc }}$ are computed. The normalization is such that the diagonal elements of $\hat{\mathbf{A}}$ are unity. If the feed elements are identical, then under our assumption that the isolated element patterns closely approximate the embedded element patterns, $P_{m}=P_{1}$ for all $m$.

In order to minimize spillover noise and direct-path interference reception at the feed, restricting the array pattern in some way is desirable. One simple approach would be to back the array with a ground plane, although this would likely reduce the useful system bandwidth. We assume here that the element patterns are hemispherical but otherwise neglect effects of a backing ground plane. More detailed design aspects of the feed can easily be added to the framework presented in this paper in future studies.

Spillover noise is assumed to arrive at the feed at a solid angle extending from the reflector rim to horizontal, with the reflector pointed to zenith. The ground temperature $T_{\text {ground }}$ is set at a nominal value for the outdoor temperature $(250 \mathrm{~K})$. Atmospheric and cosmic background radiation are neglected. The pattern overlap matrix for spillover noise is defined to be

$$
\hat{A}_{\mathrm{sp}, m n}=\frac{1}{2 \eta P_{m}} \int_{\text {spill }} \bar{E}_{m}^{\mathrm{inc}{ }^{*}} \cdot \bar{E}_{n}^{\mathrm{inc}} r^{2} d \Omega
$$

where the elevation angle of the integration extends from the reflector rim to a plane perpendicular to the axis of the reflector. The covariance of the noise voltage at conjugate-matched array element feed ports can be shown to be [12]

$$
\mathbf{R}_{\mathrm{sp}}^{a}=T_{\text {ground }} \hat{\mathbf{A}}_{\mathrm{sp}}
$$

in units of Kelvin. The corresponding open-circuit covariance is

$$
\hat{\mathbf{R}}_{\mathrm{sp}}=4 R_{\mathrm{rad}} \mathbf{R}_{\mathrm{sp}}^{a}
$$

where $R_{\text {rad }}$ is the radiation resistance of one array element. In this expression, we have used the real part of the self-impedance of each element as the radiation resistance.

The vector of open circuit signal voltages at the array element feed ports is

$$
\hat{\mathbf{v}}_{s}=\frac{4 \pi \sqrt{S_{0}^{\mathrm{sig}}}}{k_{0} I_{0} \sqrt{\eta}} \mathbf{E}^{s}
$$

where $I_{0}$ is the driving current used to excite the element as a transmitter to compute $\bar{E}_{m}^{\text {inc }}$ and $k_{0}$ is the operating wavenumber. $\mathbf{E}^{s}=\left[\hat{p} \cdot \bar{E}_{1} \hat{p} \cdot \bar{E}_{2} \ldots \hat{p} \cdot \bar{E}_{N}\right]^{T}$ is a vector of phasor field strengths radiated in the direction of the signal source by the transmit array and $\hat{p}$ is the polarization of the incident field. This expression assumes that $S_{0}^{\mathrm{sig}}$ is the signal flux density in both polarizations, but that only half the signal power is received by the single-polarized feed. The open-circuit interferer response $\hat{\mathbf{v}}_{i}$ is defined similarly.

\section{B. Antenna Array and Matching Network}

Careful characterization of the impact of mutual coupling and receiver noise on the multiantenna receiving system performance requires detailed modeling of the front-end impedance matching network, LNAs, and amplifier load terminations. Fig. 1 shows a block diagram of this system. For analysis purposes, we use scattering parameters (S-parameters) referenced to a real impedance $Z_{0}$ to describe the network signals wherein 


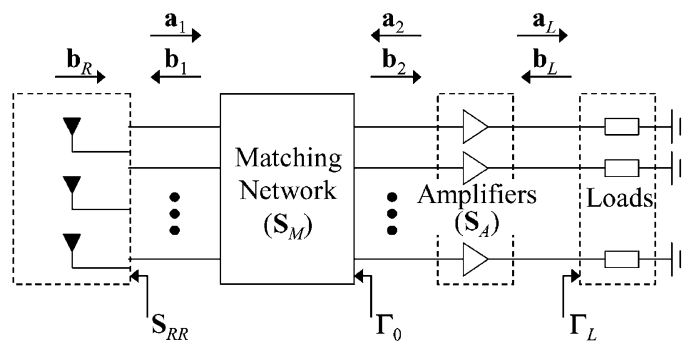

Fig. 1. Block diagram of the multiantenna receiver system including mutually coupled arrays, matching network, receiver amplifiers, and loads. After amplification, the voltages across the loads are either combined using a hardware beamformer or sampled and combined in digital signal processing.

the forward and reverse traveling waves are denoted as $\mathbf{a}$ and $\mathbf{b}$, respectively. In the following formulation, the traveling waves generically represent any of the externally supplied responses (signal, interference, or spillover noise).

The traveling waves at the receiver input satisfy

$$
\mathbf{a}_{1}=\mathbf{b}_{R}+\mathbf{S}_{R R} \mathbf{b}_{1}
$$

where $\mathbf{S}_{R R}$ represents the S-parameter matrix for the coupled antenna array. We therefore first need to relate the voltage $\hat{\mathbf{v}}$ on the open-circuit antenna ports to the source voltage wave $\mathbf{b}_{R}$. Since $\mathbf{a}_{1}=\mathbf{b}_{1}$ for an open-circuit termination, (9) gives $\mathbf{b}_{R}=$ $\left(\mathbf{I}-\mathbf{S}_{R R}\right) \mathbf{a}_{1}$. We also recognize that $\hat{\mathbf{v}}=Z_{0}^{1 / 2}\left(\mathbf{a}_{1}+\mathbf{b}_{1}\right)=$ $2 Z_{0}^{1 / 2} \mathbf{a}_{1}$, which upon substitution into our expression for $\mathbf{b}_{R}$ yields

$$
\mathbf{b}_{R}=\frac{1}{2 Z_{0}^{1 / 2}}\left(\mathbf{I}-\mathbf{S}_{R R}\right) \hat{\mathbf{v}}
$$

The matching network in Fig. 1 provides a proper impedance interface between the antenna and LNAs. The multiport nature of the receiver suggests representing the matching network using a block matrix S-parameter description, or

$$
\mathbf{S}_{M}=\left[\begin{array}{ll}
\mathbf{S}_{11} & \mathbf{S}_{12} \\
\mathbf{S}_{21} & \mathbf{S}_{22}
\end{array}\right]
$$

where 1 and 2 refer to input and output ports, respectively. Since $\mathbf{b}_{1}=\mathbf{S}_{11} \mathbf{a}_{1}+\mathbf{S}_{12} \mathbf{a}_{2}$ and $\mathbf{b}_{2}=\mathbf{S}_{21} \mathbf{a}_{1}+\mathbf{S}_{22} \mathbf{a}_{2}$, we can use (9) to show that the signal at the matching network output is

$$
\begin{aligned}
\mathbf{b}_{2} & =\underbrace{\mathbf{S}_{21}\left(\mathbf{I}-\mathbf{S}_{R R} \mathbf{S}_{11}\right)^{-1}}_{\mathbf{G}} \mathbf{b}_{R}+\boldsymbol{\Gamma}_{0} \mathbf{a}_{2} \\
\boldsymbol{\Gamma}_{0} & =\mathbf{S}_{22}+\mathbf{G} \mathbf{S}_{R R} \mathbf{S}_{12}
\end{aligned}
$$

where $\Gamma_{0}$ represents the reflection coefficient at the matching network output (see Fig. 1).

\section{Noisy Amplifier Output Signal}

The bulk of the receiver thermal noise is created in the front-end LNAs. To maintain tractability in the analysis, we will consider amplifier topologies that can be described by input-output S-parameters and standard noise parameters. The $m$ th amplifier injects forward and reverse traveling noise waves $a_{\eta, m}$ and $b_{\eta, m}$, respectively, at the amplifier input [34]. Using the notation of Fig. 1 coupled with the conventions in [34], the amplifier signal plus noise output waves are of the form

$$
\begin{aligned}
\mathbf{a}_{2} & =\mathbf{S}_{A, 11} \mathbf{b}_{2}+\mathbf{S}_{A, 12} \mathbf{b}_{L}-\mathbf{S}_{A, 11} \mathbf{a}_{\eta}+\mathbf{b}_{\eta} \\
\mathbf{a}_{L} & =\mathbf{S}_{A, 21} \mathbf{b}_{2}+\mathbf{S}_{A, 22} \mathbf{b}_{L}-\mathbf{S}_{A, 21} \mathbf{a}_{\eta}
\end{aligned}
$$

where the S-parameters $\mathbf{S}_{A, i j}$ describe the amplifiers. Using these expressions with (10) and (12) along with the relation $\mathbf{b}_{L}=\Gamma_{L} \mathbf{a}_{L}$, the voltage across the amplifier terminations $\mathbf{v}_{L}=$ $Z_{0}^{1 / 2}\left(\mathbf{I}+\boldsymbol{\Gamma}_{L}\right) \mathbf{a}_{L}$ becomes

$$
\mathbf{v}_{L}=\mathbf{Q}\left[\mathbf{G}_{0} \hat{\mathbf{v}}+\mathbf{\Gamma}_{0} \mathbf{b}_{\eta}-\mathbf{a}_{\eta}\right]
$$

where

$$
\begin{aligned}
\mathbf{G}_{0}= & \frac{1}{2 Z_{0}^{1 / 2}} \mathbf{G}\left(\mathbf{I}-\mathbf{S}_{R R}\right) \\
\mathbf{Q}= & Z_{0}^{1 / 2}\left(\mathbf{I}+\boldsymbol{\Gamma}_{L}\right)\left[\left(\mathbf{I}-\boldsymbol{\Gamma}_{0} \mathbf{S}_{A, 11}\right) \mathbf{S}_{A, 21}^{-1}\left(\mathbf{I}-\mathbf{S}_{A, 22} \boldsymbol{\Gamma}_{L}\right)\right. \\
& \left.-\boldsymbol{\Gamma}_{0} \mathbf{S}_{A, 12} \boldsymbol{\Gamma}_{L}\right]^{-1}
\end{aligned}
$$

This development now allows us to transform the signals at the open-circuit antenna terminals to those across the amplifier loads as

$$
\begin{aligned}
\text { Signal: } \mathbf{v}_{s} & =\mathbf{Q G}_{0} \hat{\mathbf{v}}_{s} \\
\text { Interferer: } \mathbf{v}_{i} & =\mathbf{Q G}_{0} \hat{\mathbf{v}}_{i} \\
\text { Spillover Noise: } \mathbf{R}_{\mathrm{sp}} & =\mathbf{Q}^{*} \mathbf{G}_{0}^{*} \hat{\mathbf{R}}_{\mathrm{sp}} \mathbf{G}_{0}^{T} \mathbf{Q}^{T} \\
\text { Overlap Matrix: } \mathbf{A} & =\mathbf{Q}^{*} \mathbf{G}_{0}^{*} \hat{\mathbf{A}} \mathbf{G}_{0}^{T} \mathbf{Q}^{T}
\end{aligned}
$$

If we assume that the noise in each amplifier is uncorrelated with that of all other amplifiers, then we can write

$$
\begin{aligned}
\mathrm{E}\left\{\mathbf{a}_{\eta} \mathbf{a}_{\eta}^{\dagger}\right\} & =T_{\alpha} \mathbf{I} \\
\mathrm{E}\left\{\mathbf{b}_{\eta} \mathbf{b}_{\eta}^{\dagger}\right\} & =T_{\beta} \mathbf{I} \\
\mathrm{E}\left\{\mathbf{a}_{\eta} \mathbf{b}_{\eta}^{\dagger}\right\} & =T_{\gamma}^{*} \mathbf{I}
\end{aligned}
$$

in units of $\mathrm{K}$, where $\mathrm{E}\{\cdot\}$ denotes an expectation and $T_{\alpha}, T_{\beta}$, and $T_{\gamma}$ are amplifier effective noise temperatures [34]. The superscript $\dagger$ denotes the conjugate transpose operation. The covariance of the thermal noise injected by the LNAs is, therefore

$$
\begin{aligned}
\mathbf{R}_{\mathrm{LNA}} & =\mathbf{Q}^{*} \underbrace{\mathrm{E}\left(\boldsymbol{\Gamma}_{0} \mathbf{b}_{\eta}-\mathbf{a}_{\eta}\right)^{*}\left(\boldsymbol{\Gamma}_{0} \mathbf{b}_{\eta}-\mathbf{a}_{\eta}\right)^{T}}_{\mathbf{R}_{\eta}} \mathbf{Q}^{T} \\
\mathbf{R}_{\eta} & =T_{\alpha} \mathbf{I}+T_{\beta} \boldsymbol{\Gamma}_{0}^{*} \boldsymbol{\Gamma}_{0}^{T}-T_{\gamma}^{*} \boldsymbol{\Gamma}_{0}^{*}-T_{\gamma} \boldsymbol{\Gamma}_{0}^{T}
\end{aligned}
$$

\section{Matching Network Specification}

The amplifier noise figures are minimized when there is a balance between signal power coupling from the array elements to the amplifiers and cancellation of the forward and reflected reverse amplifier noise waveforms. We conjecture that this is achieved when the array input reflection coefficient looking from the amplifier inputs into the matching network is $\boldsymbol{\Gamma}_{0}=\Gamma_{\mathrm{opt}} \mathbf{I}$, where $\Gamma_{\mathrm{opt}}$ is the input reflection coefficient that maximizes SNR for the single amplifier case. The value of $\Gamma_{\mathrm{opt}}$ is determined by the amplifier specifications. A general 
proof that this particular input reflection coefficient maximizes output SNR for the array case has not yet been obtained. But it is possible to demonstrate numerically that it is optimal for a given system. This can be done by using (19)-(22), (32), and (37) to show that for a given steering angle, the maximum attainable SNR is

$$
\mathrm{SNR}_{\max }=\hat{\mathbf{v}}_{s}^{T} \underbrace{\mathbf{G}_{0}^{T}\left(\mathbf{R}_{\eta}+\mathbf{G}_{0}^{*} \hat{\mathbf{R}}_{\mathrm{sp}} \mathbf{G}_{0}^{T}\right)^{-1} \mathbf{G}^{*}}_{\mathbf{M}\left(\Gamma_{0}\right)} \hat{\mathbf{v}}_{s}^{*} .
$$

This expression implies that the SNR is a linear combination of the singular values of the matrix $\mathbf{M}$. For a given set of array and amplifier parameters, the singular values of $\mathbf{M}\left(\boldsymbol{\Gamma}_{0}\right)$ were computed numerically for a set of matrices $\boldsymbol{\Gamma}_{0}$ which spanned the space of realizable reflections for reciprocal matching networks without gain. The singular values were largest for $\Gamma_{0}=\Gamma_{\text {opt }} \mathbf{I}$, so the SNR for all possible steered beam directions must be smaller for any other choice of $\boldsymbol{\Gamma}_{0}$. This procedure was used for several different choices of array and amplifier specifications, and in all cases considered, $\boldsymbol{\Gamma}_{0}=\Gamma_{\text {opt }} \mathbf{I}$ was the optimal match. This provides strong empirical evidence that $\boldsymbol{\Gamma}_{0}=\Gamma_{\text {opt }} \mathbf{I}$ is the optimal match in general.

Therefore, we will specify $\boldsymbol{\Gamma}_{0}=\Gamma_{\text {opt }} \mathbf{I}$ and determine the matching network subblocks $\mathbf{S}_{i j}$ required to achieve this condition. To begin, we take the singular value decomposition (SVD) of each subblock to obtain $\mathbf{S}_{i j}=\mathbf{U}_{i j} \boldsymbol{\Lambda}_{i j}^{1 / 2} \mathbf{V}_{i j}^{\dagger}$. Assuming that the matching network is lossless $\left(\mathbf{S}_{M}^{\dagger} \mathbf{S}_{M}=\mathbf{I}\right)$ and reciprocal $\left(\mathbf{S}_{M}=\mathbf{S}_{M}^{T}\right)$, it can be shown that the subblocks can be written as

$$
\begin{aligned}
& \mathbf{S}_{11}=\mathbf{U}_{11} \boldsymbol{\Lambda}_{11}^{1 / 2} \mathbf{U}_{11}^{T} \\
& \mathbf{S}_{12}=j \mathbf{U}_{11}\left(\mathbf{I}-\boldsymbol{\Lambda}_{11}\right)^{1 / 2} \mathbf{U}_{22}^{T} \\
& \mathbf{S}_{21}=j \mathbf{U}_{22}\left(\mathbf{I}-\boldsymbol{\Lambda}_{11}\right)^{1 / 2} \mathbf{U}_{11}^{T} \\
& \mathbf{S}_{22}=\mathbf{U}_{22} \boldsymbol{\Lambda}_{11}^{1 / 2} \mathbf{U}_{22}^{T} .
\end{aligned}
$$

where $\boldsymbol{\Lambda}_{i j}^{1 / 2}$ is a diagonal matrix of the singular values of $\mathbf{S}_{i j}$.

With $\boldsymbol{\Gamma}_{0}=\mathbf{U}_{0} \boldsymbol{\Lambda}_{0}^{1 / 2} \mathbf{U}_{0}^{T}$ representing the SVD of $\boldsymbol{\Gamma}_{0}$, we use (13) and (27) to obtain

$$
\begin{aligned}
\boldsymbol{\Gamma}_{0} & =\mathbf{U}_{22}\left[\boldsymbol{\Lambda}_{11}^{1 / 2}-\left(\mathbf{I}-\boldsymbol{\Lambda}_{11}\right)^{1 / 2} \mathbf{T}\left(\mathbf{I}-\boldsymbol{\Lambda}_{11}\right)^{1 / 2}\right] \mathbf{U}_{22}^{T} \\
\mathbf{T} & =\mathbf{U}_{11}^{T}\left(\mathbf{I}-\mathbf{S}_{R R} \mathbf{U}_{11} \boldsymbol{\Lambda}_{11}^{1 / 2} \mathbf{U}_{11}^{T}\right)^{-1} \mathbf{S}_{R R} \mathbf{U}_{11}
\end{aligned}
$$

We have flexibility in specifying the singular vectors $\mathbf{U}_{11}$ and $\mathbf{U}_{22}$ and therefore choose representations that lead to mathematical simplicity. First, we see that if $\mathbf{S}_{R R}=\mathbf{U}_{R R} \boldsymbol{\Lambda}_{R R}^{1 / 2} \mathbf{U}_{R R}^{T}$, then by choosing $\mathbf{U}_{11}=\mathbf{U}_{R R}^{*}$ we obtain

$$
\mathbf{T}=\left(\mathbf{I}-\boldsymbol{\Lambda}_{R R}^{1 / 2} \boldsymbol{\Lambda}_{11}^{1 / 2}\right)^{-1} \boldsymbol{\Lambda}_{R R}^{1 / 2}
$$

which is diagonal. If we further choose $\mathbf{U}_{22}=\mathbf{U}_{0}$, we can solve (28) to obtain

$$
\boldsymbol{\Lambda}_{11}^{1 / 2}=\left(\boldsymbol{\Lambda}_{0}^{1 / 2}+\boldsymbol{\Lambda}_{R}^{1 / 2}\right)\left(\mathbf{I}+\boldsymbol{\Lambda}_{0}^{1 / 2} \boldsymbol{\Lambda}_{R}^{1 / 2}\right)^{-1}
$$

Achieving diagonal $\boldsymbol{\Gamma}_{0}$ in general requires a coupled matching network to "undo" the coupling created by the antenna. It is however common to assume that the coupled antenna can be represented using the diagonal elements of the full impedance matrix $\mathbf{Z}_{R R}$ describing the array, leading to a diagonal $\overline{\mathbf{S}}_{R R}$ with elements $\bar{S}_{R R, i i}=\left(Z_{R R, i i}-Z_{0}\right) /\left(Z_{R R, i i}+Z_{0}\right)$. This value of $\overline{\mathbf{S}}_{R R}$ is then used in place of $\mathbf{S}_{R R}$ to specify the singular vectors and singular values as outlined above, leading to an uncoupled matching network. However, when analyzing the performance of such a match, the complete nondiagonal form of $\mathbf{S}_{R R}$ must be used in the analysis (16). We refer to this suboptimal diagonal network as a self-impedance match.

\section{E. Beamformers for Optimal SNR and SINR}

After amplification by the front-end LNAs, the $N$ received signals are combined using the complex beamformer weights $\mathbf{w}=\left[\begin{array}{llll}w_{1} & w_{2} & \ldots & w_{N}\end{array}\right]^{T}$. The output SNR can be expressed as

$$
\mathrm{SNR}=\frac{\mathbf{w}^{\dagger} \mathbf{R}_{s} \mathbf{w}}{\mathbf{w}^{\dagger} \mathbf{R}_{n} \mathbf{w}}
$$

where the total thermal noise covariance is $\mathbf{R}_{n}=\mathbf{R}_{\mathrm{sp}}+\mathbf{R}_{\mathrm{LNA}}$ and the signal covariance is

$$
\mathbf{R}_{s}=\mathbf{v}_{s}^{*} \mathbf{v}_{s}^{T}
$$

It is assumed that all covariances are normalized to power $/ \mathrm{Hz}$ in units of Kelvin. The thermal noise and interferer covariance matrix is

$$
\mathbf{R}_{N}=\mathbf{R}_{\mathrm{sp}}+\mathbf{R}_{\mathrm{LNA}}+\mathbf{R}_{i}
$$

For a single plane wave interferer signal, $\mathbf{R}_{i}$ has the form of (33) but with the fields evaluated in the direction of the interferer. For multiple uncorrelated interferers, $\mathbf{R}_{i}$ can be taken to be the sum of single plane wave correlations. The SINR is

$$
\mathrm{SINR}=\frac{\mathbf{w}^{\dagger} \mathbf{R}_{s} \mathbf{w}}{\mathbf{w}^{\dagger} \mathbf{R}_{N} \mathbf{w}} .
$$

The maximum-SNR beamformer is determined by the generalized eigenvalue problem

$$
\mathbf{R}_{s} \mathbf{w}=\lambda_{\max } \mathbf{R}_{n} \mathbf{w}
$$

where $\lambda_{\max }$ is the generalized eigenvalue with largest magnitude. If the noise covariance matrix is nonsingular, the max-SNR weights reduce to

$$
\mathbf{w}_{\mathrm{SNR}, \max }=\mathbf{R}_{n}^{-1} \mathbf{E}^{s^{*}} \text {. }
$$

If an interfering signal is included in the model, then the noise covariance matrix in (36) and (37) is replaced with the signal and interferer covariance $\mathbf{R}_{N}$.

\section{NUMERICAL RESULTS}

\section{A. Simulation Parameters}

Feed array elements are located on a hexagonal grid in the $x-y$ plane (Fig. 2). The physical spacing between elements is fixed 


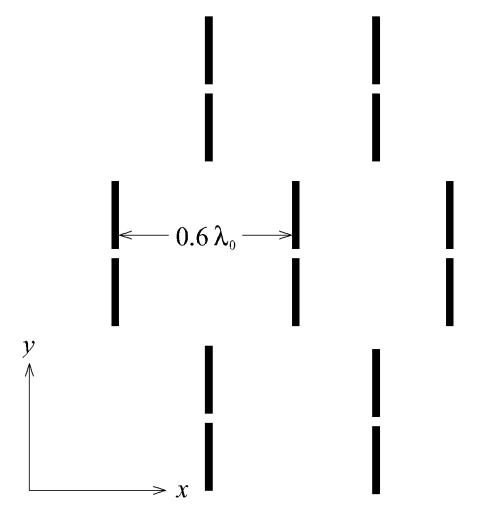

Fig. 2. Focal plane array element locations and orientations.

at 0.6 wavelengths $(11.2 \mathrm{~cm})$ at the center frequency $f_{0}=1.6$ $\mathrm{GHz}$. The array elements are $y$-directed thin-wire dipoles and located such that one of the elements lies at $x=0.6 \lambda_{0}$ relative to the center element. The dipole lengths are $0.5 \lambda_{0}$. Dipoles were chosen because closed form approximations are available for the mutual impedances [35]. The radiation patterns of the array elements were assumed to be hemispherical in computing received spillover noise. The reflector is a $D=25 \mathrm{~m}$ diameter paraboloid with $f / D=0.36$, where $f$ is the focal length of the reflector. Relative to the feed, the center of the reflector lies at $z=-f$.

As described in the previous section, the radiation patterns of each array element with the others open-circuited are approximated by the isolated element pattern, which is available in closed form. For small element spacings, this approximation breaks down, potentially leading to such abberations as more available power at the element terminals than is incident on the reflector. In order to obtain the mutual impedances of the dipole elements, a sinusoidal approximation for the current on the antennas was used [35]. This model also becomes inaccurate for small element spacings, leading to eigenvalues of the impedance matrix with a negative real part, indicating a nonphysical gain for reverse-traveling waves reflected at the antenna ports. We expect perturbations due to these approximations to be neglible for spacings above, say, $0.3 \lambda$.

To compute radiated fields for the array and reflector system, we employ the efficient PO-based line integral method of [36]. This approach requires that an approximation be made in the azimuthal dependence of the dipole radiation pattern. A scale factor is included so that the reflector is effectively illuminated by the correct total power. As this scaling only becomes significant for large electrical dipole lengths, the effect on numerical results over the useful bandwidth is small. Multiple scattering between the feed and the reflector is neglected. In order to overcome these simplifying approximations, a method of moments simulation could be employed to model the combined array and reflector system [37].

The signal is a single plane wave arriving at $0^{\circ}$ relative to the reflector axis. The flux density of the signal is $1 \mathrm{Jy}=10^{-26}$ $\mathrm{W} / \mathrm{m}^{2} / \mathrm{Hz}$. The performance of an array feed with respect to off-axis beam steering angle has been considered in [12]. Since the feed elements are polarized and the signal is assumed unpolarized, half of the incident power is received. With this choice of signal intensity, SNR is equivalent to the system sensitivity $G / T_{\text {sys }}$ if $G$ is expressed in units of $\mathrm{K} / \mathrm{Jy}$.

The interferer is a point source at an angle of arrival of $30^{\circ}$ relative to the reflector axis (boresight), and an azimuth angle of $0^{\circ}$ relative to the coordinate system of the feed array. The interferer flux density is $100 \mathrm{~dB}$ greater than the signal flux density. At the center frequency, the interference to spillover noise ratio (INR) at the feed port of the center element is approximately 10 $\mathrm{dB}$. As a function of frequency the INR fluctuates because the sidelobe pattern of the reflector changes.

As a model LNA, a transistor with S-parameters $S_{11}=0.942 \angle-40.7^{\circ}, S_{12}=0.033 \angle 61.6^{\circ}, S_{21}=$ $5.401 \angle 140.7^{\circ}, S_{22}=0.523 \angle-35.0^{\circ}$ at the center frequency $(1.6 \mathrm{GHz})$ is chosen. The minimum noise figure is $F_{\min }=0.5$ $\mathrm{dB}$ for the optimal source termination of $\Gamma_{\mathrm{opt}}=0.86 \angle 31^{\circ}$, and the noise resistance is $R_{n}=9.5 \Omega$. The ambient temperature is assumed to be that of liquid nitrogen $(77 \mathrm{~K})$, so that the equivalent noise temperature in terms of the forward traveling noise signal at the LNA input port is nominally $2.4 \mathrm{~K}$. To avoid complicating biases in the results, the transistor parameters are taken to be constant as a function of frequency, which is not unreasonable since available transistors can have relatively stable parameters over a large bandwidth.

\section{B. Matching Network Cases}

We consider several types of matching networks, described here in order of decreasing optimality.

1) No Mutual Coupling, Infinite Bandwidth Optimal Match: Without mutual coupling, the optimal matching network reduces to a diagonal network which couples one array element to only one amplifier. For this case, the matching network is optimized at each frequency point, corresponding to an ideal, unrealizable "infinite bandwidth" matching network, which provides a performance bound for any realized matching network.

2) Mutual Coupling, Infinite Bandwidth Optimal Match: If the effects of mutual coupling are included, the optimal array-toamplifier matching network becomes a nondiagonal, fully coupled multiport network. The network is optimized at each frequency.

3) Mutual Coupling, Infinite Bandwidth Self-Impedance Match: In order to reduce the complexity of the full multiport matching network, a suboptimal, diagonal network which couples one array element to one amplifier can be employed. As with the preceding cases, this simpler network is optimized at each frequency.

4) Mutual Coupling, Quarter-Wavelength Line and Reactance: The array-to-amplifier matching networks assumed in the preceding cases are unrealizable, because they require a different physical matching network at each frequency in the operating bandwidth. These idealized matches are nevertheless of interest, because they provide upper bounds on performance and allow the effects of mutual coupling on the received signal power and spillover noise to be isolated from degradation of the noise performance of the amplifiers.

In order to gain insight into system performance with a realizable network, we consider the case of a quarter wave impedance transformer and reactance at each amplifier input. 


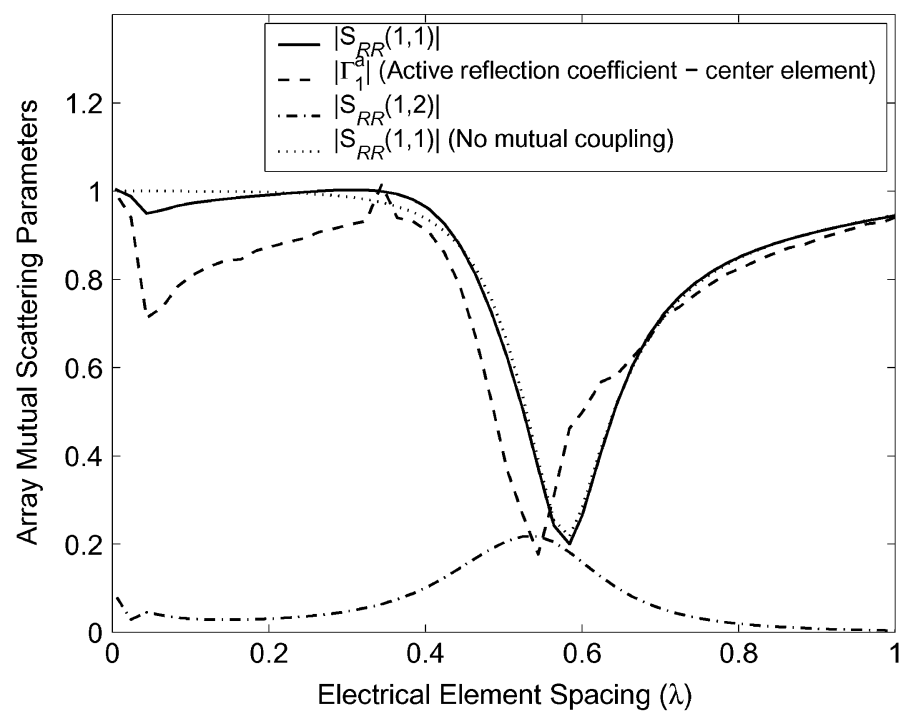

Fig. 3. Array mutual scattering parameters and active reflection coefficient as a function of frequency. The self-coupling of the center element and the mutual coupling between the center element and one of the outer elements are shown. The narrow width of the region for which $\left|\mathbf{S}_{R R}(1,1)\right|$ is much smaller than unity limits the usable system bandwidth for a simple matching network. The physical array element spacing is fixed, and the electrical element spacing in wavelengths changes as a function of frequency, so the horizontal axis is a normalized frequency scale.

A simple matching network is also important for low noise applications, because the loss introduced by a complicated network could lead to an intolerable increase in the system thermal noise temperature. This network is nonideal both in its frequency response and in that it is not cross-coupled, so mutual coupling effects are neglected in the matching network design. The network is designed at the center frequency $(1.6 \mathrm{GHz}, 0.6 \lambda$ element spacing). Variation of the array mutual impedances with frequency leads to degraded system performance away from the center frequency.

5) Mutual Coupling, No Matching Network: As a baseline case, we also include the case of no matching network at all $\left(\mathbf{S}_{11}=\mathbf{S}_{22}=\mathbf{I}, \mathbf{S}_{12}=\mathbf{S}_{21}=0\right)$. In this case, system performance is determined arbitrarily by the amplifier input impedance.

\section{Array and Matching Network Scattering Parameters}

In order to provide a feel for how rapidly the array element and matching network impedances change with frequency, representative scattering parameters are shown in Figs. 3 and 4. All $\mathrm{S}$-parameters are relative to a system impedance of $Z_{0}=50 \Omega$. It can be seen that the self-impedance of the array elements is a limiting factor in determining system bandwidth, because $\mathbf{S}_{R R}(1,1)$ changes rapidly near the center frequency.

In Fig. 3, the magnitude of the active reflection coefficient of the center array element is also shown. The active impedance of the $n$th element is

$$
Z_{n}^{a}=\frac{\hat{\mathbf{v}}_{n}}{\left[\mathbf{Z}_{R R}^{-1} \hat{\mathbf{v}}\right]_{n}}
$$

which can be obtained from the equivalent circuit model described in [38]. The open-circuit excitation is taken to be $\hat{\mathbf{v}}=$ $\hat{\mathbf{v}}_{s}+\hat{\mathbf{v}}_{i}$, although the effect of the interfering signal on the ac-

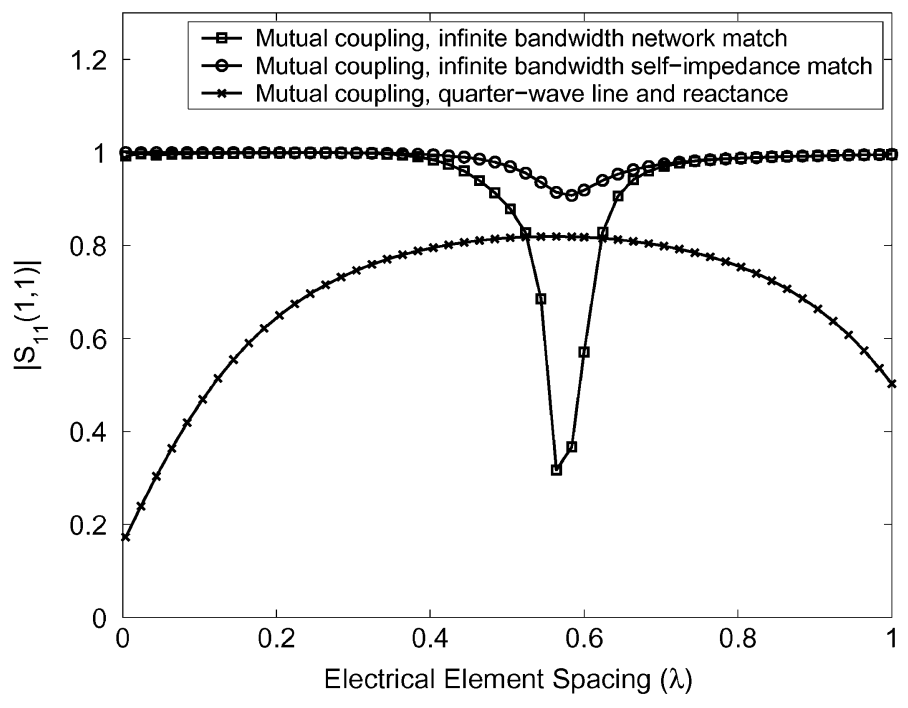

Fig. 4. Scattering parameters of array-to-amplifier matching network for the port connected to the center element. The simple quarter-wave line and reactance matching network does not have enough degrees of freedom to follow the optimal response over a broad band.

tive impedance is very small. The active reflection coefficient is $\Gamma_{n}^{a}=\left(Z_{n}^{a}-Z_{0}\right) /\left(Z_{n}^{a}+Z_{0}\right)$. It can be seen that the active reflection coefficient of the center element is similar to the 1,1 element of the array mutual scattering matrix.

Fig. 4 shows that only the broadband matching networks can follow the rapidly changing array self-impedance. The quarterwave line and reactance have a smoother frequency response, which deviates from ideal more rapidly as frequency changes, so this simpler matching network leads to a narrower system bandwidth. It is interesting to note that while it might be expected that the self-impedance match and the quarter-wave with reactance would lead to identical S-parameters at the center frequency, the realized S-parameters are different, because a two-port matching network with constrained output reflection coefficient for a given input impedance is nonunique.

\section{Array Quality Factor}

For closely spaced arrays, optimal gain solutions may enter a superdirectivity regime, which is undesirable since this results in large sensitivity of directivity to small fluctuations in array element weights. The quality factor of the feed is a measure of the degree of superdirectivity. The $Q$ of the array system at the output port of the beamformer is given approximately by the geometrical quality factor [39], [40]

$$
Q_{g}=\frac{\mathbf{w}^{\dagger} \mathbf{w}}{\mathbf{w}^{\dagger} \mathbf{A} \mathbf{w}}
$$

multiplied by the $Q$ of a single isolated element, which is typically on the order of unity. The matching network at the element feed ports also contributes to the overall quality factor of the system, but we consider here only the geometrical $Q$-factor given by (39). In order to remove the scaling effects of the matching network and amplifier gain, we take the array $Q$-factor to be defined at the element output ports, so that

$$
Q_{g}=\frac{\hat{\mathbf{w}}^{\dagger} \hat{\mathbf{w}}}{\hat{\mathbf{w}}^{\dagger} \hat{\mathbf{A}} \hat{\mathbf{w}}}
$$




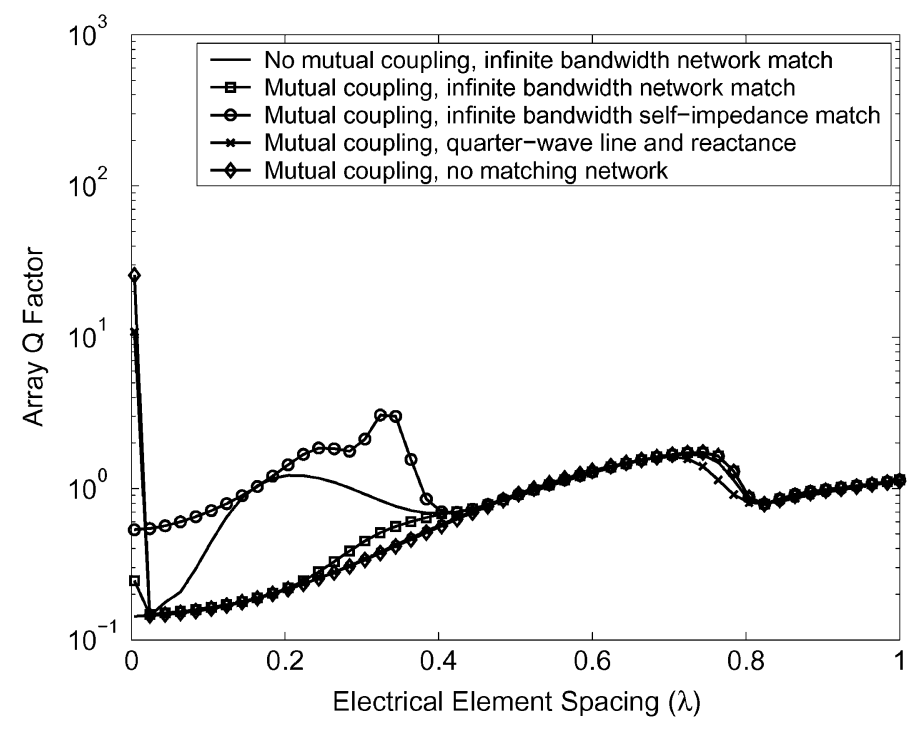

Fig. 5. Array quality factor for the no-interfererer case.

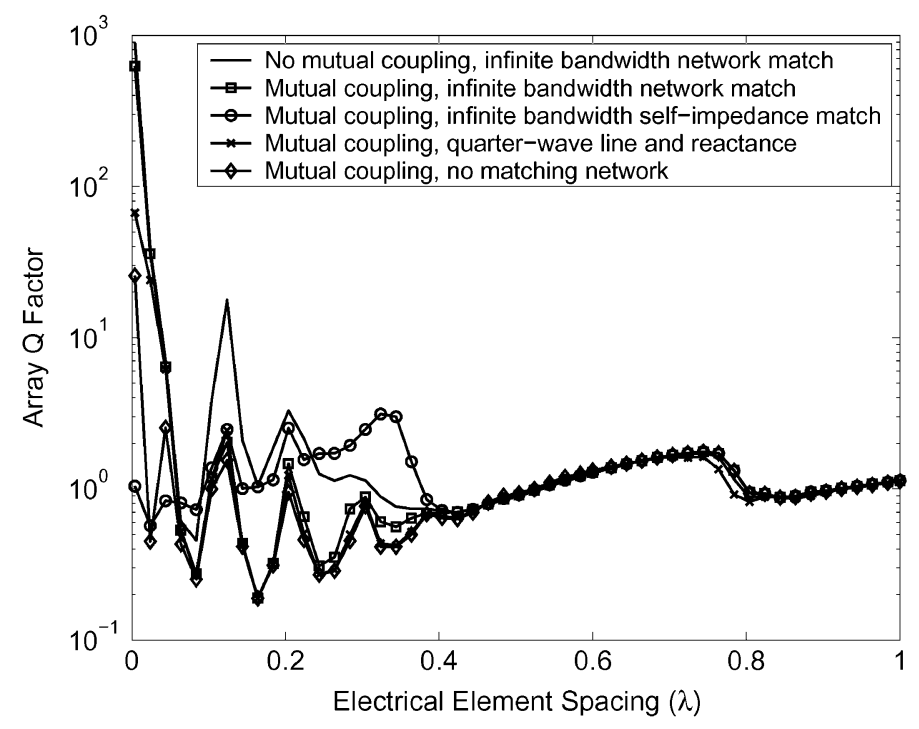

Fig. 6. Array quality factor with an interfering signal.

where $\hat{\mathbf{w}}=\mathbf{G}_{0}^{T} \mathbf{Q}^{T} \mathbf{w}$. Although it is difficult to make a more quantitative statement, a $Q$-factor of order unity indicates that the array is not operating in the superdirectivity regime.

The array $Q$-factors for each of the matching networks are shown in Figs. 5 and 6 . The $Q$-factor is influenced by the matching network and amplifier parameters, but as can be seen from (40) it is primarily a function of the beamformer weights. The $Q$-factor becomes large at the low frequencies, where the SNR performance of the array begins to degrade. Over the useful bandwidth, the $Q$-factor is acceptably low. The effect of the interfering signal is to produce beamformer solutions with larger $Q$-factor at low frequencies.

\section{E. System Sensitivity}

For the no-interferer case, system sensitivity is shown in Fig. 7. In the absence of mutual coupling, higher overall system sensitivity can be attained than is possible if the effects of mutual coupling are included. Generally, mutual coupling

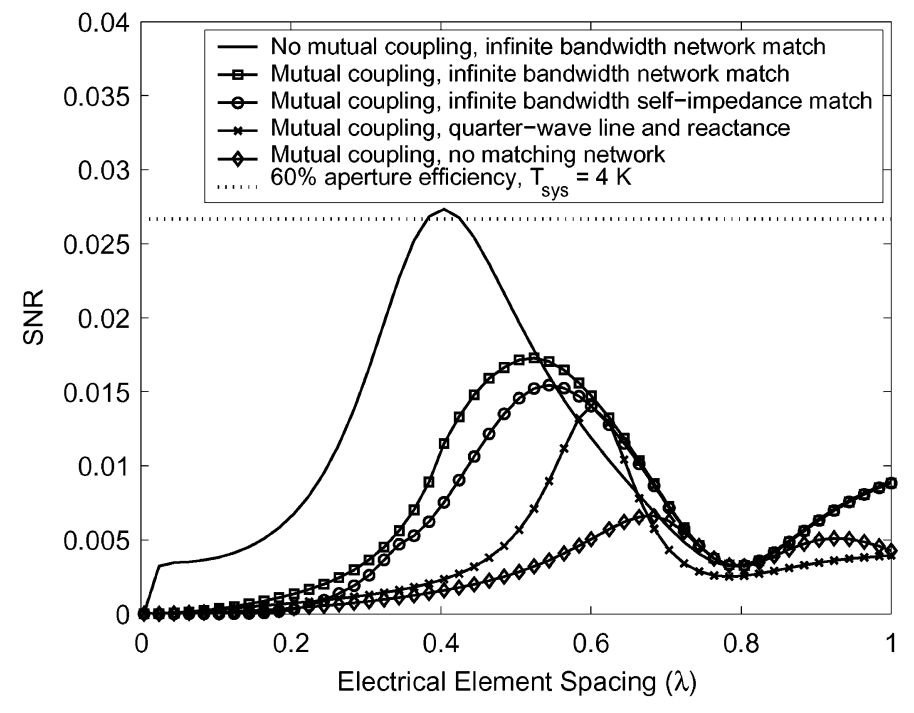

Fig. 7. Output SNR in the no-interferer case for several types of array-to-amplifier matching networks. The physical array element spacing is fixed, and the electrical element spacing in wavelengths changes as a function of frequency.

reduces the sensitivity, except over a small band near the center frequency. At very low frequencies, the sensitivity without mutual coupling is likely nonphysically large, because the overlap between the effective aperture areas of the array elements is not taken into account. From a network point of view, a strongly coupled mutual impedance matrix leads to low power coupling from the array elements to the amplifier input ports. For reference, the sensitivity

$$
\mathrm{SNR}_{\mathrm{ref}}=\frac{\eta_{\mathrm{ap}} A S_{0}^{\mathrm{sig}}}{2 k_{B} T_{\mathrm{sys}}}=0.027
$$

where $A$ is the physical area of the reflector and $k_{B}$ is Boltzman's constant, for an aperture efficiency of $\eta_{\mathrm{ap}}=60 \%$ and a system noise temperature of $T_{\mathrm{sys}}=4 \mathrm{~K}$ is also shown on these figures.

Although the infinite bandwidth self-impedance match neglects mutual coupling in the matching network design, performance is close to that of the idealized full network match. In both cases, the $3 \mathrm{~dB}$ bandwidth is $50 \%(0.8 \mathrm{GHz})$. This is significant, because a fully coupled multiport match would be much more difficult to realize in practice than simple two-port matching networks between each element and one amplifier. The quarter wave with reactance case leads to a narrower useful bandwidth (23\%, or $0.37 \mathrm{GHz})$, because of the constrained frequency response of the network.

It can be seen in Fig. 8 that performance is not significantly degraded by the presence of an interfering signal. Of great importance is the stability of the system gain and sensitivity as the spatial filter adapts to changes in the interferer direction of arrival or intensity. As the frequency is swept, the reflector response pattern changes, so the interferer response moves through the sidelobes of the pattern much as if it were moving in location at a fixed operating frequency. This leads to rapid changes in the received interferer power levels at the array elements, which in turn affects the beamformer solution and causes variations in the system sensitivity with frequency. Fig. 8 shows 


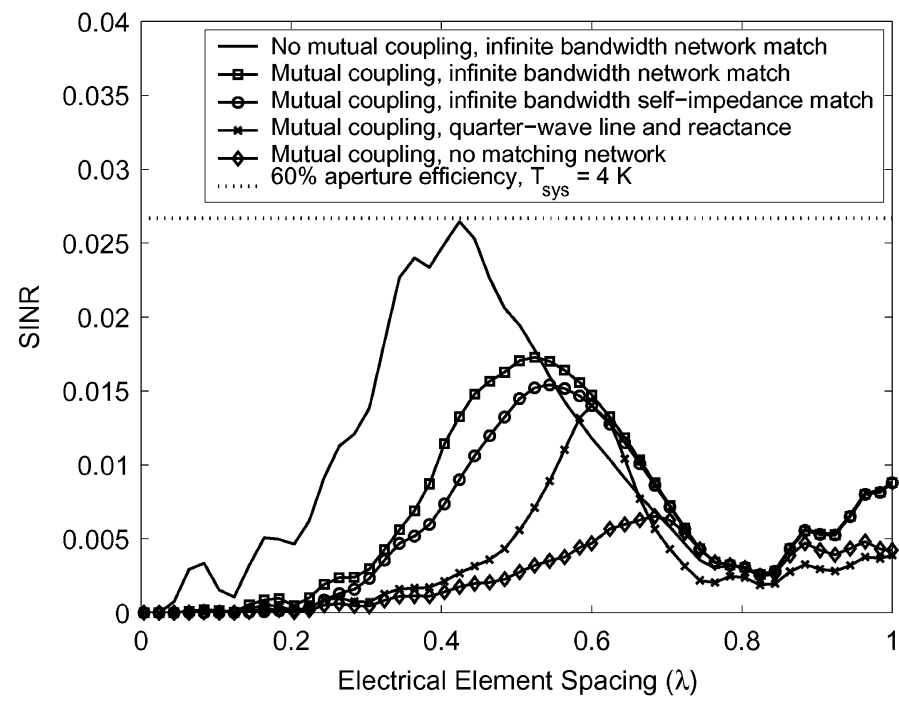

Fig. 8. Output SINR with an intefering signal for several types of array-to-amplifier matching networks. Fluctuations at high and low frequencies are pattern rumble caused by the frequency dependence of the antenna response pattern in the interferer direction.

that near the center frequency, the perturbation in sensitivity due to this effect is small. For the optimal matching network, the variation relative to the SNR is at most $2.5 \%(0.1 \mathrm{~dB})$ over the $3 \mathrm{~dB}$ bandwidth. Away from the center frequency, the changing received interferer power levels have a greater effect on system sensitivity, leading to significant pattern rumble. This occurs for all of the matching networks, although the pattern rumble is smaller for the mutually coupled case than for an uncoupled array. Thus, the usable system bandwidth is limited not only by rolloff of the overall sensitivity away from the center frequency, but also by increased susceptibility to pattern rumble.

\section{CONCLUSION}

For a focal plane array feed, we have studied the effects of mutual coupling and the array-to-amplifier matching network on system sensitivity. The matching network affects sensitivity through the amount of signal power coupled from the array element terminals to the amplifiers, and by presenting an effective load at the amplifier inputs which determines the degree of cancellation between partially correlated forward and reverse thermal noise signals generated by the amplifiers. After the matching network and amplifiers, signals were combined using a beamformer for maximum ouput SNR.

The design criterion for the array to amplifier matching network was minimum amplifier noise figures. Realistic matching networks are suboptimal in two respects: 1) the optimal matching network for a mutually coupled array requires cross-coupling between all input and output ports, whereas a practical network would likely have a simpler topology, and 2) the frequency response of a realized network would only be close to that of the optimal solution for a given topology over a limited bandwidth. With respect to network topology, numerical results indicated that a simpler, diagonal self-impedance matching network yields substantially the same performance as the optimal full network match. With respect to frequency response, an unrealizable, "infinite bandwidth" matching network can compensate to a degree for the rapidly varying input impedances of the dipole array elements. But for a realistic network with a constrained frequency response, the matching network can only transform the antenna impedances to be close to the optimal impedance at the amplifier inputs for minimum noise figure over a narrow band. This is evident in degraded system performance for the case of a quarter-wave transmission line section and reactance between each array element and amplifier.

Simulations of the output signal to noise ratio were given both with and without interference. Because the reflector sidelobe pattern changes with frequency, the array response to the interferer and the beamformer solution also change, leading to pattern rumble. Because the relative variability increases away from the center frequency, this effect also limits usable system bandwidth for applications requiring high gain stability. The pattern variability was smaller for a mutually coupled array than for an uncoupled array.

With a larger array, additional outer elements serve primarily to receive signal power that would miss the feed if it were smaller. For this reason, we expect that performance would be qualitatively similar for a larger array, but with improved overall sensitivity. As far as the influence of the amplifier noise parameters, for an ideal or near-ideal matching network, the amplifier noise figure is close to optimal, and the total output noise power is dominated by the amplifier noise contribution, so sensitivity would be decreased by a larger amplifier noise figure.

In future work, it would be desirable to employ a method of moment simulation to obtain the open-circuit loaded array radiation patterns, rather than approximating the open-circuit patterns by the isolated element pattern. Such a simulation would allow for inclusion of a backing ground plane, and would enable investigation of more sophisticated antenna elements with improved bandwidth for which closed-form approximations for mutual impedances are not available.

\section{REFERENCES}

[1] C. Barnbaum and R. F. Bradley, "A new approach to interference excision in radio astronomy: Real-time adaptive cancellation," Astronomical J., vol. 116, pp. 2598-2614, Nov. 1998.

[2] S. W. Ellingson, J. D. Bunton, and J. F. Bell, "Removal of the GLONASS $\mathrm{C} / \mathrm{A}$ signal from $\mathrm{OH}$ spectral line observations using a parametric modeling technique," The Astrophysical J. Supplement Series, no. 135, pp. 87-93, Jul. 2001.

[3] A. J. Poulsen, B. Jeffs, K. Warnick, and R. Fisher, "Real-time adaptive cancellation of GLONASS interference in OH signal observations at the green bank telescope," presented at the IEEE APS-URSI Symp., Columbus, OH, Jun. 23-27, 2003.

[4] B. Smolders and G. Hampson, "Deterministic RF nulling in phased arrays for the next generation of radio telescopes," IEEE Antennas Propagat. Mag., vol. 44, no. 8, pp. 13-22, Aug. 2002.

[5] J. Raza, A.-J. Boonstra, and A.-J. van der Veen, "Spatial filtering of RF interference in radio astronomy," IEEE Signal Process. Lett., vol. 9, pp. 64-67, Feb. 2002.

[6] S. Ellingson and G. Hampson, "A subspace-tracking approach to interference nulling for phased array-based radio telescopes," IEEE Trans. Antennas Propag., vol. 50, no. 1, pp. 25-30, Jan. 2002.

[7] S. Ellingson, "Beamforming and interference canceling with very large wideband arrays," IEEE Trans. Antennas Propag., vol. 51, no. 6, pp. 1338-1346, Jun. 2003. 
[8] B. D. Jeffs, L. Li, and K. F. Warnick, "Auxiliary assisted interference mitigation for radio astronomy arrays," IEEE Trans. Signal Process., vol. 52, no. 2, pp. 439-451, Feb. 2005, to be published.

[9] J. G. Bij de Vaate and G. W. Kant, "The phased array approach to SKA, results of a demonstrator project," presented at the Eur. Microwave Conf., Milan, Italy, Sep. 23-24, 2002.

[10] G. C. Bower, "A radio frequency interference mitigation strategy for the Allen telescope array," presented at the URSI General Assembly XXVII, Aug. 17-24, 2002.

[11] J. F. Bell, P. J. Hall, R. J. Sault, and L. Kewley. (2000, Aug.) Implementing interference suppression: Impacts on SKA system design. Technological Pathways to the SKA [Online]. Available: http://www.jb.man.ac.uk/ska/workshop/Bell2.pdf

[12] C. K. Hansen, K. F. Warnick, B. D. Jeffs, J. R. Fisher, and R. Bradley, "Interference mitigation using a focal plane array," Radio Sci., 2005, to be published.

[13] P. Shelton, "Multiple-feed systems for objectives," IEEE Trans. Antennas Propag., vol. 13, no. 6, pp. 992-994, Nov. 1965.

[14] A. W. Rudge and M. J. Withers, "New techniques for beam steering with fixed parabolic reflector," Proc. Inst. Elect. Eng., vol. 118, pp. 857-863, Jul. 1971.

[15] T. S. Bird, J. L. Boomars, and P. J. B. Clarricoats, "Multiple-beam dualoffset reflector antenna with an array feed," Electron. Lett., vol. 14, no. 4, pp. 439-441, Jul. 1978.

[16] S. J. Blank and W. A. Imbriale, "Array feed synthesis for correction of reflector distortion and vernier beamsteering," IEEE Trans. Antennas Propag., vol. AP-36, no. 10, pp. 1351-1358, Oct. 1988.

[17] J. A. Murphy and R. Padman, "Focal-plane and aperture-plane heterodyne array receivers for millimeter-wave radioastronomy-A comparison," Int. J. Infrared Millimeter Waves, vol. 9, pp. 667-704, Aug. 1988.

[18] D. Emerson and J. Payne, Eds., Astronomical Soc. Pacific Conference Series, Multi-Feed Systems for Radio Telescopes. San Francisco, CA: Astronomical Society of the Pacific, 1995, vol. 75.

[19] L. Stavely-Smith, W. E. Wilson, T. S. Bird, M. J. Disney, R. D. Ekers, K. C. Freeman, R. F. Haynes, M. W. Sinclair, R. A. Vaile, R. L. Webster, and A. E. Wright, "The Parkes $21 \mathrm{~cm}$ multibeam receiver," Publ. Astron. Soc. Aust., vol. 13, no. 3, pp. 243-248, 1996.

[20] J. R. Fisher. (1996, Sep.) Phased Array Feeds for Low Noise Reflector Antennas. [Online]. Available: http://www.gb.nrao.edu/rfisher/Papers/ af_ieee.ps

[21] M. Ivashina and C. van't Klooster, "Focal fields in reflector antennas and associated array feed synthesis for high efficiency multi-beam performances," presented at the ESTEC Antenna Workshop, Noordwijk, The Netherlands, Sep. 18-20, 2002.

[22] J. R. Fisher, "Phased Array Feeds for Low Noise Reflector Antennas," NRAO, Green Bank, WV, Tech. rep., Jan. 30, 1996.

[23] J. R. Fisher and R. F. Bradley, "Technical progress on array feeds and RFI cancellation," presented at the Int. Square-Kilometer Array Science Meeting, Calgary, Canada, Jul. 19-22, 1998.

[24] J. R. Fisher, R. F. Bradley, R. Escoffer, and K. Saini, "Phased array feed design and prototype," presented at the URSI General Assembly, Lille, France, Aug. 28-Sep. 51996.

[25] R. F. Bradley, K. Saini, and J. R. Fisher, "A prototype array feed-Design and construction," presented at the Proc. USNC/URSI Meeting, Boulder, CO, Jan. 9-12, 1996.

[26] J. R. Fisher and R. F. Bradley, "Full sampling array feeds for radio telescopes," in Proc. SPIE Int. Symp. Astronomical Telescopes and Instrumentation, vol. 4015, 2000, pp. 308-319.

[27] S. Stein, "On cross coupling in multiple-beam antennas," IRE Trans. Antennas Propag., vol. 10, pp. 548-557, Sep. 1962.

[28] T. S. Bird, "Mutual coupling in arrays of coaxial waveguides and horns," IEEE Trans. Antennas Propag., vol. 52, no. 3, pp. 821-829, Mar. 2004.

[29] D. Schaubert, "Wideband Vivaldi arrays for large aperture antennas," presented at the Workshop on Perspectives on Radio Astronomy: Technologies for Large Antenna Arrays, The Netherlands, Apr. 12-14, 1999.

[30] A. Moumen and L. P. Ligthart, "Synthesis of a dielectric rod array for a multiple beam reflector antenna," in Proc. Antennas and Propagation Soc. Int. Symp., vol. 4, Jul. 2000, pp. 1914-1917.

[31] T. Straumann and G. Moench, "Effects of mutual antenna coupling on SAIR performance," in Proc. 8th Int. Conf. Antennas and Propagation, vol. 1, 1993, pp. 532-535.

[32] M. Jensen and J. Wallace, "Analysis of coupling in multi-antenna communication systems," in IEICE Trans., vol. E87-C, Sep. 2004, pp. $1418-1424$.
[33] C. Craeye, B. Parvais, and X. Dardenne, "MoM simulation of signal-tonoise patterns in infinite and finite receiving arrays," IEEE Trans. Antennas Propag., vol. 52, no. 12, pp. 3245-3256, Dec. 2004.

[34] J. Engberg and T. Larsen, Noise Theory of Linear and Nonlinear Circuits. New York: Wiley, 1995.

[35] C. A. Balanis, Antenna Theory: Analysis and Design. New York: Wiley, 1997.

[36] W. A. Imbriale, P. G. Ingerson, and W. C. Wong, "Large lateral feed displacements in a parabolic reflector," IEEE Trans. Antennas Propag., vol. AP-22, no. 6, pp. 742-745, Nov. 1974.

[37] A. Moldsvor and P. Kildal, "Systematic approach to control feed scattering and multiple reflections in symmetrical primary-fed reflector antennas," Proc. Inst. Elect. Eng., pt. H, vol. 139, pp. 65-71, Feb. 1992.

[38] J. D. Reale, "The receive voltage transmission function of a phased array antenna element," IEEE Trans. Antennas Propag., vol. 25, no. 4, pp. 542-547, Jul. 1977.

[39] M. Uzsoky and L. Solymar, "Theory of super-directive linear arrays," Acta Phys. Acad. Sci. Hung., vol. 6, p. 195, 1956.

[40] Y. T. Lo, S. W. Lee, and Q. H. Lee, "Optimization of directivity and signal-to-noise ratio of an arbitrary antenna array," Proc. IEEE, vol. 54, pp. 1033-1045, Aug. 1966.

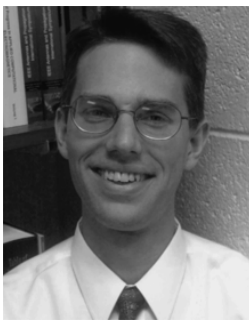

Karl F. Warnick (S'95-M'98-SM'04) received the B.S. degree (magna cum laude) with University Honors and the Ph.D. degree from Brigham Young University (BYU), Provo, UT, in 1994 and 1997, respectively.

From 1998 to 2000, he was a Postdoctoral Research Associate and Visiting Assistant Professor in the Center for Computational Electromagnetics at the University of Illinois at Urbana-Champaign. Since 2000, he has been an Assistant Professor in the Department of Electrical and Computer Engineering at BYU. He has coauthored a book chapter and over 50 conference presentations and scientific journal papers. Research interests include computational electromagnetics, rough surface scattering, remote sensing, inverse scattering, and numerical analysis.

Dr. Warnick was a recipient of the National Science Foundation Graduate Research Fellowship.

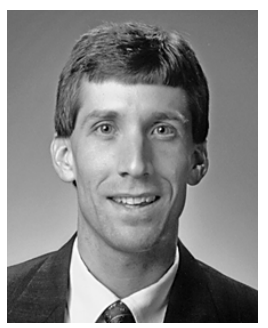

Michael A. Jensen (S'93-M'95-SM'01) received the B.S. (summa cum laude) and M.S. degrees in electrical engineering from Brigham Young University (BYU), Provo, UT, in 1990 and 1991, respectively, and the Ph.D. degree in electrical engineering from the University of California, Los Angeles (UCLA), in 1994

From 1989 to 1991, he was a Graduate Research Assistant in the Lasers and Optics Laboratory, BYU. From 1991 to 1994 , he was a Graduate Student Researcher in the Antenna Laboratory, UCLA. Since 1994, he has been at the Electrical and Computer Engineering Department, BYU, where he is currently an Associate Professor. His main research interests include antennas and propagation for personal communications, microwave circuit design, radar remote sensing, numerical electromagnetics, and optical fiber communications.

Dr. Jensen is a Member of Eta Kappa Nu and Tau Beta Pi. In 1990, he received a National Science Foundation Graduate Fellowship. He was awarded the best student paper award at the 1994 IEEE International Symposium on Antennas and Propagation, and received the Harold A. Wheeler Applications Prize Paper Award in the IEEE TRANSACTIONS ON ANTENNAS AND PROPAGATION in 2002. He currently serves on the Joint Meetings Committee for the IEEE Antennas and Propagation Society and as an Associate Editor for the IEEE TRANSACTIONS on ANTENNAS AND PROPAGATION. He was the Technical Program Chair for the 2000 IEEE International Symposium on Antennas and Propagation and the General Co-Chair for the IEEE Topical Conference on Wireless Communications in 2003. 